\title{
When can cultural selection explain adaptation?
}

\author{
Azita Chellappoo ${ }^{1,2}$ (1)
}

Received: 17 August 2020 / Accepted: 21 December 2021 / Published online: 3 January 2022

(c) The Author(s) 2022

\begin{abstract}
Cultural selection models aim to explain cultural phenomena as the products of a selective process, often characterising institutions, practices, norms or behaviours as adaptations. I argue that a lack of attention has been paid to the explanatory power of cultural selection frameworks. Arguments for cultural selection frequently depend on demonstrating only that selection models can in principle be applied to culture, rather than explicitly demonstrating the explanatory payoffs that could arise from their application. Understanding when and how cultural selection generates powerful explanations is crucial to evaluating cultural selection, as well as realising its promised epistemic and practical benefits. I argue that the ability for cultural selection to explain 'design without a designer' is crucial to successful and powerful cultural selection explanations. I introduce the strategy of comparing cultural selection to goal-directed agent accounts in order to evaluate when cultural selection can provide distinctive explanatory payoffs, drawing on two case studies to illustrate the benefits of this strategy. I argue that a focus on phenomena which cannot be explained through intention or agency-based explanations in particular could provide a fruitful avenue to identifying the cases where cultural selection can be insightfully applied.
\end{abstract}

Keywords Cultural evolution · Cultural selection · Explanation

\section{Introduction}

Evolution by natural selection has transformed our understanding of the biological world, providing a powerful explanation for the range of adaptations we see in biological organisms. Many aspects of culture seem similarly adaptive, in that they benefit individuals or groups, and allow us to thrive in a range of environments.

Azita Chellappoo

azita.chellappoo@open.ac.uk

1 Institut Für Philosophie I, Ruhr-Universität Bochum, Universitätsstr. 150, 44801 Bochum, Germany

2 Present Address: Department of Philosophy, Faculty of Arts and Social Sciences, The Open University, Walton Hall, Milton Keynes MK7 6AA, UK 
Joseph Henrich (2015) gives the example of the Pama-Nyungan peoples in Australia: various aspects of their culture, including their ritual and foraging practices, were adaptive in the sense that they enabled the maintenance of large communities. In large-scale industrial societies, we could view a range of institutions as having adaptive value: for example, the cultural norms of firms may contribute to their success on the marketplace, conservation laws may be adaptive in that they ensure the long-term sustainability of a particular resource, and adherence to social norms such as driving on the same side of the road allow large communities to coordinate their actions. Given this range of apparently adaptive behaviour, the application of selection to culture appears promising.

Cultural selection is a thriving area of research that often aims to characterize cultural phenomena, including institutions, practices, norms and behaviours, as adaptations arising from a selective process. Broadly, we can understand cultural selection as referring to "selection on differences between individuals with respect to their cultural variants" (Birch 2017, p. 196). ${ }^{1}$ Cultural selection has been invoked as an explanation for a diverse range of phenomena, including the evolution of ethnic markers (McElreath et al. 2003), the development of prosocial religion (Norenzayan et al. 2016), adaptive food taboos (Henrich and Henrich 2010), large-scale human warfare (Zefferman and Mathew 2015), and the broad cooperative norms and institutions that characterise human societies (Richerson and Boyd 2005). Authors such as Henrich (2015) make the case that the success of the human species in thriving in a vast range of environments is due to our unique capacity for cumulative cultural evolution. He draws upon examples of failed European colonial expeditions, such as that of the explorers Burke and Wills, to show how important the cumulative cultural adaptations built up by indigenous people were to their survival. The fact that Europeans struggled to survive in those same environments, despite having the same innate cognitive capacities, is taken as an indication that it is the lack of cumulative cultural adaptations that determined their failure, rather than, for example, the kinds of mental modules posited by evolutionary psychologists. Richerson and Boyd (2005) similarly stress the power of cultural selection to build cultural adaptations. Their work has included the development of formal models to show that, given certain assumptions regarding cultural transmission and inheritance, beneficial norms could spread through populations through selection (e.g., Boyd and Richerson 2002, 2009; Boyd et al. 2011).

Cultural selection research has often centered around the capacity for cultural selection to operate in principle, relying on drawing analogies between cultural and biological systems (e.g., Mesoudi et al. 2004; Richerson et al. 2016), or developing formal models that demonstrate that cultural selection mechanisms could in principle drive the spread of adaptive variants (e.g., Boyd and Richerson 2009). Cultural

\footnotetext{
${ }^{1}$ Birch (2017, p. 197-202) goes on to distinguish between two types of cultural selection: one where differences in cultural variants result in differences in biological fitness (which can also occur in nonhuman species) and one where differences in cultural variants result in differences in cultural fitness. A significant proportion of work in the field concerns the second type (particularly studies exploring recent or current cultural selection), and this is what I primarily focus on here.
} 
selection can be understood as an analogue of natural selection, where cumulative selection could lead to complex cultural adaptations. Cultural selection can be couched in terms of the Lewontin conditions: there is variation in cultural traits, these traits differ in their fitness, and there is a mechanism of inheritance (Lewontin 1970). ${ }^{2}$ If these conditions are fulfilled, we would expect fitter traits to spread throughout the population. When this process is cumulative, we could see the generation of complex adaptations resulting from successive rounds of selection.

If the claims of cultural selection proponents were true, then cultural selection could be a powerful force in understanding many aspects of cultural phenomena: both the origin of universal tendencies that have shaped human societies over millennia, as well as the development of key social institutions that structure human interaction, and ongoing changes in current norms and practices.

However, the explanatory value of cultural selection has been under-attended to. The capacity for cultural selection to act in principle does not mean it is meaningfully explanatory in practice. In assessing the merits of using cultural selection frameworks or models, it is important not just to show that cultural systems possess features necessary to undergo adaptive evolution, but also to identify the explanatory payoffs that cultural selection can give us, over and above alternative frameworks. Despite claims that progress in the social sciences has been painfully slow (Mesoudi et al. 2006), it is undeniable that there already exists a very substantial body of work in disciplines, such as anthropology, sociology, and history, that study cultural phenomena and cultural change, using their own frameworks and methodologies, and with their own explanatory goals. To some extent, given the range of work already in existence, the challenge for advocates of cultural selection is to show that there are significant aspects of culture still lacking explanation, that these lack explanation due to the shortcomings of other frameworks, and that an evolutionary approach can make meaningful contributions to our understanding of these phenomena.

Furthermore, this question becomes particularly important when considering the practical benefits that cultural selection frameworks have been touted to bring. It has been suggested that applying cultural selection models could be impactful in fields such as sustainability science and innovation. In fact, some authors have asserted that evolutionary principles should be at the centre of redesigning worldviews, institutions, and technologies (Beddoe et al. 2009). If cultural selection is going to be able to bring these meaningful benefits in terms of addressing urgent global challenges, it must be clear both whether and to what extent cultural selection can explain the relevant phenomena.

I focus on one epistemic good that cultural selection can bring: the ability to explain 'design without a designer'. I argue that this a key component of successful and powerful cultural selection explanations. One way to determine when cultural selection explanations are likely to be either plausible or powerful is to compare cultural selection with a goal-directed agent account: if a goal-directed agent account

\footnotetext{
${ }^{2}$ Cutural selection has also been couched in terms of the Price equation (e.g., El Mouden et al. 2014), viewing cultural selection in the second sense described by Birch (2017), i.e. as the maximisation of cultural fitness.
} 
can explain everything that a cultural selection account can, this indicates that cultural selection is not a useful tool in this case.

In section 'Explaining 'Design without a Designer' I argue for the claim that the explanation of 'design without a designer' is a central feature of cultural selection. Although there are other epistemic goods that arise from cultural selection, I contend that explaining phenomena that a goal-directed agent account struggles to explain is central to the explanatory power of cultural selection. In section 'Contrasting cultural selection and goal-directed agent explanations' I introduce and defend the strategy of comparing a goal-directed agent explanation with a cultural selection explanation. In section 'Case studies' I consider two case studies that illustrate the benefits of this comparative strategy: the application of cultural selection in the context of sustainability science, and the characterisation of cultural adaptations in small-scale societies. I then consider two objections to this strategy in section 'Objections'.

\section{Explaining ‘Design without a Designer'}

Darwin's theory of evolution by natural selection has been phenomenally successful in providing a unifying framework, bringing together insights from a wide range of disciplines (physiology, development, and taxonomy, amongst others), and transforming our understanding of the biological world. Given this success, it is therefore tempting to attempt to expand the application of Darwin's theory to domains outside of biology. In fact, this is the explicit motivation given by some cultural evolution theorists for the cultural evolution project as a whole. For example, Mesoudi and colleagues in a 2006 paper articulate the promise of the application of Darwinian theory to culture as the creation of a 'cultural synthesis' that mirrors what has been achieved in biology, with what they see as disparate and poorly understood cultural phenomena becoming unified under a broad evolutionary approach. Similarly, Muthukrishna and Henrich (2019, p. 223) suggest that dual inheritance theory could function as "a theory of human behaviour to unify the psychological and behavioural sciences".

One way in which the debates over cultural selection have unfolded is in terms of the resemblance between cultural and natural selection. There are debates over whether cultural selection should be considered Darwinian or Lamarckian, due to phenomena such as guided variation (Kronfeldner 2007). ${ }^{3}$ Additionally, as has been

\footnotetext{
3 There are ongoing debates regarding the extent to which cultural selection requires blind variation, often in the context of divisions between cultural evolution researchers who emphasise either the role of cultural selection or the role of transformation and attraction in patterns of cultural change or stasis (Acerbi and Mesoudi 2015). Some argue that the importance of cultural selection depends on the extent to which variation is 'blind', rather than 'guided' (e.g., Claidière et al, 2018), and argue that, since cultural variation is often guided to a large degree, selection is rarely an important force. 'Guided' variation could occur through cognition, or through the environment: this refers to a range of processes that systematically bias the variation that arises in a population. However, others argue that this distinction is less crucial: for example, Henrich et al. (2008, p. 129) argue that "there is no doubt that, as people acquire and modify beliefs, ideas, and values, the variation that is generated can be highly non-random, and these nonselective processes shape cultural variation. But so what? Selection occurs anytime there is heritable variation that affects survival or reproduction (transmission). It does not matter whether the
} 
noted by many authors, key concepts in cultural selection do not have systematic definitions or solid theoretical grounding, such as cultural fitness, or the units of cultural selection (Wimsatt 1999; Crozier 2008). It is certainly the case that cultural systems bear many disanalogies with biological systems. However, I contend that focusing on the explanatory aptness or power of cultural selection provides a more fruitful avenue in assessing cultural selection frameworks compared to these debates about the strength of the analogy between culture and biology (although these debates have illuminated many important points of consideration).

Confusion or vagueness around core concepts in selection is not unique to culture: we see similar debates in the context of evolutionary biology over the appropriate fitness concept, or population concept, for example (Ariew and Ernst 2009; Stegenga 2014), or rethinking the necessity of reproduction, taken to be a core feature of populations that can undergo selection, as in Papale (2020). The lack of a single fitness or population concept has not hampered the capacity for natural selection to be a powerful explanation for organismal adaptation, amongst other phenomena, suggesting that this should not be a barrier for the application of cultural selection. For example, Ramsey and De Block (2017) note that cultural fitness concepts have real and substantial differences from biological fitness concepts. Nevertheless, they argue that we can build a concept of cultural fitness that can do the necessary work in feeding into cultural selection explanations.

I therefore focus on the explanatory payoffs of cultural selection: why are we applying it? What can we explain that we could not previously? For biological organisms, there are clear reasons to invoke natural selection. Darwin's theory of evolution by natural selection provided a naturalistic explanation for the phenomenon of organisms appearing adapted to their environment, with a range of ingenious solutions to problems of survival (i.e., the appearance of design without the existence of a designer). One key aspect of the explanatory power of natural selection lies in its capacity to explain how features of organisms that appear designed to deal with specific challenges posed by their environment came about, without invoking intelligence or purpose within that explanation. As Nettle (2020, p. 2) notes, "the key insight of Darwinian genetic evolutionary theory was that design-like properties could be produced, over time, by selection processes. Thus, it is quite natural, seeing

\footnotetext{
Footnote 3 (continued)

variation is random". Similarly, Mesoudi et al. (2004, p. 4) suggest that "although this variation may not be entirely random with respect to selection, ultimately it matters less to the Darwinian process how variation arises, than that variation exists and is exposed to selection". My argument here is not directly connected to these debates, in that I am not aiming to give a general account of the conditions under which cultural selection occurs. If one believes that cultural selection is rarely important, on the basis of the prevalence of guided variation, then one will already be convinced that cultural selection explanations are often inappropriate or do not provide epistemic benefits. However, if one takes the position of Henrich or Mesoudi, then there will be many cases in which the conditions for cultural selection to operate will obtain. In this case, we can still ask the question of whether there are explanatory benefits to applying cultural selection. Additionally, there may be cases where variation is blind (and so both sides of this debate would agree that cultural selection could, in principle, be at play), and yet, cultural selection does not provide explanatory benefits over and above a goal-directed agent account.
} 
design-like properties in culture, to assume they must be produced by selection processes too".

I argue that we should expect cultural selection frameworks to be able to do the same, explaining adaptive fit between cultural variants and environment without invoking intention or agency. Although selection plays other explanatory roles in biological systems, one distinctive role is that which it plays in explaining adaptation, or design. I use 'adaptation' as synonymous with 'design' here, adopting the definition given by Wertz and Moya (2019), in a paper which highlights the role that 'design' plays in cultural evolutionary theories, as a "structure that is well-suited to solve a problem". As previously noted, we see 'adaptive' cultural traits, or those with the appearance of 'design', everywhere around us, if we construe 'adaptive' or 'designed' in this broad sense. This is the role that has been highlighted by several key figures in the cultural selection literature: cultural selection proponents often point to the capacity for cultural selection to explain 'cultural adaptations' (Richerson and Boyd 2005; Henrich and Henrich 2010; Boyd et al. 2011; Norenzayan et al. 2016; Kline et al. 2018). These adaptations, construed broadly as structures well-suited to solving a problem, could be at either the individual or the group level. Additionally, I use this broad definition to avoid assuming the truth of any particular account (cultural selection or intentional design) from the outset. ${ }^{4}$

I will first clarify what is meant here by 'explanation' and 'explanatory power'. I will then suggest that, while cultural selection accounts often are able to provide explanations of cultural phenomena, they sometimes lack explanatory power in comparison to other approaches. I defend this claim against two possible responses: that cultural selection can be explanatorily powerful even when it does not explain 'design without a designer' and that cultural selection can still be a useful framework even if it is not explanatorily powerful. I will elaborate on the strategy of comparing cultural selection to a goal-directed agent account in section 'Contrasting cultural selection and goal-directed agent explanations'.

\section{Explanation and explanatory power}

Although it is beyond the scope of this paper to explore in detail and defend a particular philosophical account of explanation, it is worth clarifying what it might mean for a cultural selection explanation to truly explain, or to be explanatorily powerful. There are two related considerations: firstly, whether a particular account constitutes a successful or adequate explanation, and secondly, the degree to which an explanation has explanatory power.

There are many accounts of scientific explanation; I will discuss briefly two of the most prominent. Under a causal account (such as Woodward 2005, 2010), broadly, an explanation involves making a counterfactual claim about the relationship

\footnotetext{
4 A narrower definition, such as one that defines an adaptive trait in terms of the extent to which it allows an individual or group to survive (and perhaps reproduce), or one that defines an adaptive trait in terms of the fulfilment of a goal or intention, would be problematic insofar as it assumes the truth or utility of either cultural selection or a goal-directed agent account from the outset.
} 
between cause and effect: changing the cause would bring about a change in effect. ${ }^{5}$ Giving a causal explanation for a phenomenon is to specify the causal structure that is responsible for the production of the outcome. Therefore, to characterise an adaptive trait as the result of cultural selection would be to identify a selective process as the cause that is in some way responsible for the adaptive trait. This could be the distribution of the trait in the population (why the trait is widespread, or why some groups have this trait and not others), or the origin of the trait itself (understood in the sense outlined in Godfrey-Smith 2009, where successive rounds of selection can explain why a trait exists in its current form at all). In many of the cases that are characterised as instances of cultural selection, the intervention that would change the cause and search for a change in effect will have to be hypothetical, as, for example, in cultural group selection interpretations of the development of prosocial tendencies in early human societies. The construction of mathematical models provides one way in which these hypothetical interventions can be explored. However, there is a significant strand of experimental work which implements reallife interventions to study the relative impacts of selection and other features of cultural transmission (e.g., Acerbi and Tehrani 2018).

Under a unificationist account (such as Kitcher 1981), explanation means unifying a range of different phenomena. This is something that has clear relevance to cultural selection: authors have explicitly advocated for the ability for evolutionary approaches to culture to unify disparate cultural phenomena (Mesoudi et al. 2006). It certainly seems to be the case that cultural selection has more potential to unify phenomena than (for example) historical explanations, which may be highly local and contextual. If a range of apparently disconnected and very different cultural traits, such as food taboos in Fiji, incest avoidance practices, conservation laws, and the appeal of Kim Kardashian, could be explained through reference to the same process (selection), then under a unificationist account cultural selection seems like a successful explanation. However, it is important to be careful here: Kitcher's account requires not only range (that the theory pertains to explananda across many domains) but also stringency. The stringency criterion means that explanations must also be restricted, and therefore be able to rule out explananda. This is to prevent explanations being vacuous. I suggest this is a tendency we see in the literature (made clearer by the case studies in section 'Objections'): cultural selection has been applied in a multitude of contexts, but often at the expense of stringency.

The debates over the similarities or differences between cultural and biological systems have an important bearing on whether cultural selection can be considered an explanation at all. If it were the case that cultural selection cannot even get off the ground, i.e., that cultural systems are too dissimilar to biological systems in key respects, then clearly we can rule them out as explanations from the start. This kind of move has been made in the debate over memetics: critics have challenged the

\footnotetext{
5 There are many dimensions to causal-interventionist accounts that provide criteria under which such an explanation is successful, including proportionality, specificity and stability. It could be a fruitful avenue for further research to consider how cultural selection explanations generally score on these criteria, although this is outside the scope of this paper.
} 
conceptual underpinnings of the research programme, such that we have prima facie reasons to dismiss memetic explanations out of hand, without consideration of particular cases or applications (an overview of these critiques can be found in Lewens 2018).

Regardless, I contend that the more common situation is one in which cultural selection can function as an explanation. In some cases, although cultural selection can explain, it can do so only weakly. This may be the more epistemically dangerous place to be: it is not that a cultural selection account leaps out as clearly unable to furnish explanation, but rather, that it appears to provide an explanation, without offering much in the way of explanatory power.

I draw on Schupbach and Sprenger's (2011) account of explanatory power to flesh this out. The core of their account of explanatory power lies in the ability for a hypothesis to decrease the degree to which we find the explanandum surprising, or to increase the degree to which the explanandum is expected. They provide a "probabilistic logic of explanation that tells us the explanatory power of a theory (explanans) relative to some proposition (explanandum), given that that theory constitutes an explanation of that proposition" (p. 4). In the case of cultural selection, we can assume that the theory constitutes an explanation of a given phenomenon (in a causal, unificationist, or other sense), which we may often have reason to do. Nevertheless, we can assess its explanatory power, and compare this to competing alternatives.

When we consider explanations for 'design without a designer', we are assuming a highly 'surprising' explanandum. If cultural selection can decrease the extent to which 'design without a designer' is surprising, then this means this explanation has a lot of explanatory power. It certainly seems that cultural selection is capable of this: natural selection accomplishes this well, and cultural selection appears to be the right kind of mechanism that could generate this.

However, the question becomes whether the phenomenon in question is really 'design without a designer' or just 'design' (adaptive traits that could have been the product of intentional action). This is where the comparison with a goal-directed agent account comes in: if a goal-directed agent account can explain the adaptive trait, we can no longer characterise it as 'design without a designer'. If that is the case, we are analysing the explanatory power of cultural selection in relation to 'design'. In these cases cultural selection will have equal explanatory power, and perhaps less explanatory power, than goal-directed agent accounts. I suggest that if the goal-directed agent account and the cultural selection account have equal explanatory power, we should prefer the goal-directed agent account on the basis of plausibility (as elaborated on in the following section).

\section{Other roles for cultural selection}

One possible response would be to highlight the other roles that selection can play, other than explaining adaptation. For example, cultural selection could play a role in 'distribution explanations' (Neander 1995; Godfrey-Smith 2009). Perhaps this is not incompatible with an intentional, goal-directed agent account (this issue will be 
confronted in section 'Defending the comparative strategy'). Particularly in cases where we have scant data, such as when reconstructing cultural trajectories in our evolutionary past, deploying cultural selection models could usefully illuminate features of cultural change. Cultural selection models could allow us to analyse patterns in the archaeological record and differentiate them from other processes, such as drift, thus providing an explanation for the spread of (for example) a particular kind of pottery throughout a population. This applies in particular to work within evolutionary archaeology (Lipo and Madsen 2001; Shennan 2020).

Whilst this response is persuasive, my argument would still mean a restriction in explanatory scope for cultural selection. ${ }^{6}$ I do not wish to deny the usefulness of these approaches. However, as previously highlighted, a key concern of the cultural selection literature are cultural adaptations. I take the analogy with biological selection to be useful here: if natural selection were unable to explain the complex and exquisite adaptations we see in the natural world (or were able to do so with no more explanatory power than a naïve goal-directed agent account, or another account we use intuitively and successfully every day), its explanatory scope would be clearly restricted. Claims regarding, for example, selection without reproduction, have often involved the question of whether selection in particular contexts is marginal, weak, or uninteresting, which is taken to mean the incapacity to act cumulatively to produce adaptation (Dawkins 1982; Godfrey-Smith 2009, 2012; Okasha 2006). Whilst cultural selection could play valuable roles in a variety of contexts other than explaining adaptation, nevertheless, adaptation is a central explanandum. Therefore, a strategy that helps to determine the contexts in which cultural selection can explain adaptation will have utility and will impact any general claims about the explanatory scope of cultural selection.

Alternatively, one could argue that cultural selection frameworks can still have value even if they do not provide increased explanatory power. We can see debates over a somewhat parallel claim in debates regarding niche construction. Some authors have argued for the value of niche construction as a framework for understanding biological phenomena. This is not because it is impossible to explain particular biological phenomena without invoking niche construction as a separate evolutionary process: indeed, opponents point out the capacity for standard approaches to explain the targets of niche construction (Scott-Phillips et al. 2014). However, its proponents maintain that recognition of niche construction as a distinct process could lead to attending to phenomena that were not attended to previously, and the development of explanations for these. Additionally, this approach could generate further insights into phenomena that are not captured well by a standard approach (Laland and Sterelny 2006; Uller and Helanterä 2017).

This response can be decomposed into two claims about the value of the niche construction framework. Firstly, niche construction can be said to have distinct

\footnotetext{
${ }^{6}$ Here, I take 'explanatory scope' to be the range of domains to which cultural selection can apply. Given my argument, cultural selection would not apply to those cases where the explanandum is an adaptation, and where cultural selection has equal or lesser explanatory power than a goal-directed agent explanation, thereby reducing its range of domain and therefore its explanatory scope.
} 
heuristic value: it sets an agenda for research, directing researchers' attention towards particular phenomena that were previously neglected. Secondly, thinking about certain phenomena in terms of niche construction may add new dimensions to our understanding, even if explanations can be formulated in terms of the standard approach. Similarly, in the case of cultural selection, one could argue that it has heuristic value. In section 'Objections' I will directly address this claim. I suggest that this is possible and would provide justification for adopting a cultural selection approach, although there are reasons to be skeptical about its heuristic value. Additionally, one could argue that cultural selection works at a different level of explanation. The objection of different levels of explanation is discussed in section 'Objections'.

Although we would expect cultural selection to often constitute an explanation of a given cultural phenomenon, there are cases where cultural selection explanations will be weak and will not present increased explanatory power compared to alternative accounts.

\section{Contrasting cultural selection and goal-directed agent explanations}

In the previous sections I have argued that cultural selection should explain the existence of 'design without a designer' in order to be explanatorily powerful. Here I elaborate on the strategy of comparing cultural selection and goal-directed explanations: I argue that when goal-directed explanations and cultural selection explanations can both explain phenomena, and with equal explanatory power, then we should prefer the goal-directed agent explanation.

Why should this be the case? Goal-directed agent explanations are widespread in cultural systems; unlike in the biological case, we see many 'designers'. Humans are intelligent, goal-directed agents who are able to design or modify tools, artefacts, and behaviours to suit their particular purposes. Furthermore, we are often able to successfully explain aspects of our human social world in terms of this goaldirectedness. We can often predict and understand the behaviour of others around us from what we know about their access to information, capacities and goals. ${ }^{7}$ I suggest that this gives us a reason to prefer the goal-directed agent account over the cultural selection account when both have equal explanatory power. Schupbach and Sprenger's conception of explanatory power does not depend on the plausibility of an explanation: explanations can be powerful but not plausible. They provide the example of dehydration versus cyanide poisoning in explaining an individual's set of symptoms: both explanations might be equally powerful in that they equally reduce the surprisingness of the explanandum, although one might be more plausible than the other given the circumstances. Given that we use goal-directed agent

\footnotetext{
7 This is related to the concept of 'folk psychology', which pertains to the cognitive capacities to explain and predict the behaviour of others. However, a goal-directed agent account does not depend on the verdicts of any debates within the folk psychology literature in philosophy of mind, including the way it is represented in the brain and the structure of folk psychology as a theory.
} 
explanations all the time, with a long track record of success, we should take them to be more plausible. This difference in plausibility can 'break the tie' when comparing cultural selection and goal-directed agent accounts.

What does a goal-directed agent account entail? It is important to clarify that this conception does not require that an individual behaves as a perfectly rational agent, maximising their expected utility. The key to a goal-directed agent account is intention: a goal-directed agent acts with intention towards a particular goal, and their action is explicable in terms of their intentions. To reiterate the point made in the previous section: if a goal-directed agent account succeeds in explaining a given phenomena and does so 'powerfully' (it decreases the surprisingness of the explanandum), then we have a 'designer'. It is the agent's intentions that furnish an explanation.

This should not be construed as making the case for goal-directed agent explanations as the be all and end all for understanding culture. Additionally, a goal-directed account as I outline it here is a somewhat naive account of human behaviour, which is broadly defined and does not offer a full account of why an agent might have those particular goals, capacities, or dispositions that she does. This is purposeful. This is intended to be the first hurdle when considering and comparing cultural selection accounts. This is to say that if we compare the two, and find the goal-directed agent explanation cannot explain, or can explain only weakly, a given phenomenon, we should not take that as evidence that the cultural selection account is necessarily the correct, or most powerful, explanation. Perhaps an anthropological, sociological, or historical account which provides explanation not only in terms of individual agent's goals, but also institutional structures and power dynamics, will improve upon a cultural selection account. However, if a cultural selection account falls at this initial hurdle (if it cannot explain more, or more powerfully, than a goal-directed agent account), then we should doubt its usefulness.

This idea is echoed in debates over 'functional' or 'social selection' explanations in the social sciences. Cultural selection can be understood as one kind of functional explanation. Critics of functional explanations in the social sciences, such as Jon Elster and Daniel Little, argue that human behaviour is typically best explained intentionally, and that adequate functional explanations in fact draw their explanatory power from identifying the intentions that sustain a given practice or institution. Elster (1984) specifically argues that, in order for an adequate functional explanation to be constructed in the social sciences, the function of an institution or behaviour must be unintended by the actors producing it, and the causal relationship between the behaviour and its function must be unrecognised by these actors. If these conditions are not met (in addition to others) then, according to Elster, a functional explanation will fail, and an explanation in terms of intentionality will be preferable.

Although for Elster, these conditions will be rarely met, cultural selection advocates may argue that there are at least some interesting examples (such as the case of cassava processing discussed in the following section) that do fulfil these stringent conditions. Wray (2002) argues that functional explanations in the social sciences are concerned with explaining the consequences of intentional behaviour that cannot be explained through the intentions of agents, and this is where they have explanatory value. Functional explanations will be particularly good at explaining 'latent 
functions', i.e., a function that a behaviour has that is unrecognised by the individuals themselves. When motives and functions diverge an explanation solely in terms of motives or intentions will be insufficient to explain the persistence of particular practices or behaviours. Although he does not draw the connection to cultural selection or cultural group selection work, this corresponds to the kinds of cases that cultural selection advocates such as Henrich identify as particularly amenable to such an analysis.

Additionally, Wray emphasises the importance of not only offering evidence in support of a functional explanation, but also demonstrating deficiencies with alternative explanations of the same phenomena. This means drawing explicit comparisons with alternative explanations, and asking what is explained by a given functional explanation that is unexplained by, for example, rational choice theory. Showing that these competing individualist explanations give rise to unexplained anomalies is key to developing a compelling functional explanation.

\section{Defending the comparative strategy}

One way to challenge this comparative strategy would be on the basis that cultural selection and goal-directed agent explanations can be compatible and should not be considered distinct strategies that can be weighed against each other. This is related to the question of other roles that cultural selection can play outlined in section 'Other roles for cultural selection'. For example, in the cultural selection approaches proposed by Henrich, Boyd, and Richerson, social learning biases can structure cultural transmission so that adaptive cultural variants spread throughout a population. Here, individuals copy other individuals due to factors such as the prevalence of a variant within the population (conformist bias) or features of the individual who others seek to copy (model biases). This could be seen as intentional in the sense that individuals are choosing to copy a particular behaviour or model.

Here, I draw on Chellappoo (2021), where I critique prestige bias (one such social learning bias) as an explanation, contrasting it with an intentional goal-directed approach. I argue that although prestige bias could be understood as describing a population-level pattern of cultural transmission, if we do not commit to understanding prestige bias as an automatic, implicit cognitive process rather than an intentional, conscious process, we lose what is distinctive about prestige bias as an explanation for behaviour. The predictions of the prestige bias account construed broadly will not differ from the predictions of a goal-directed agent account. Within the framing of my argument here, we can understand a prestige bias account construed only in a population-level sense as having equal (or perhaps lesser) explanatory power when compared to a goal-directed agent account. ${ }^{8}$

If this critique generalises to other social learning biases, then we could question the characterisation of the social learning biases that undergird cultural selection as

\footnotetext{
8 Additionally, she compares Henrich and Henrich's (2010) prestige bias explanation of the spread of food taboos in Fiji to a structurally similar case in Cambridge to illustrate how in certain framings the goal-directed agent account will leap out as the most plausible and at least as explanatorily powerful.
} 
intentional in the relevant sense. Additionally, we can see a parallel between the two senses of prestige bias (as a population-level pattern and as one that is grounded in automatic and implicit cognition) and different senses of cultural selection (as merely a population-level pattern and as one that aims to explain 'design without a designer'). I have argued in section 'Other roles for cultural selection'. For the importance of assessing cultural selection in the second sense. The comparative strategy has value in this second sense. In the following section I make use of case studies to make the value of this strategy more concrete.

\section{Case studies}

I outline two cases where the strategy of comparing cultural selection and goaldirected agent explanations can be illuminating. The first, that of cultural selection models applied to sustainability science, illustrates the dangers of neglecting the question of the explanatory payoff of cultural selection. The second, that of the cassava processing case, shows how the comparison between cultural selection and a goal-directed agent account can provide a reason to pursue a cultural selection account.

\section{Cultural selection in sustainability science}

We see precisely how selection-based explanations can fail when focusing in on a specific domain: the application of cultural selection frameworks to sustainability science. Cultural selection could in principle help us to understand and explain the aspects of institutions that contribute or work against sustainability, and to target interventions more effectively, as outlined in several papers in the 2018 special issue of the journal Sustainability Science, titled "Applying Cultural Evolution to Sustainability Challenges". I will draw on one particular example, the application of cultural selection to the historical changes in institutions and behaviour surrounding lobstering in Maine. Waring and Acheson (2018) argue that cultural group selection has shaped the institutions and behaviours surrounding this industry and its regulators. Their aim is to explain 'institutional fit': the adaptive fit between the institutions associated with the lobstering industry and the environment. So far, this seems congruent with the targets of cultural selection; the question is whether we have 'design without a designer', or simply 'design'.

\footnotetext{
9 There is a significant literature around cultural group selection in particular, often set apart from cultural selection more generally (Smith 2020 provides a review). Cultural group selection certainly poses unique challenges that cultural selection on the individual level does not, including whether cultural groups are the right kind of entity to undergo selection (Palmer et al. 1997, Morin 2018), or if cultural groups exhibit features taken to be necessary for selection, such as reproduction (Pinker 2012). However, at least some scholars understand cultural group selection as a kind of cultural selection where groups are the units under selection, as biological group selection is often understood (Clarke and Heyes 2017). The challenges to cultural group selection made by authors such as Olivier Morin and Stephen Pinker may well be persuasive; it is outside the scope of this paper to take a stance on this. However, this can be set aside for my purposes here. As set out in section 'Explaining 'Design without a Designer', I wish to sidestep the debates over the necessary conditions for cultural selection to take place and the kinds
} 
Here, I consider one of their examples in some detail: the double gauge law. This is a law that set a maximum and minimum size for saleable lobsters, thereby protecting juveniles and large reproductive-size lobsters from being caught. According to Waring and Acheson, this is an example of a 'social dilemma'. Individuals (and harbour gangs) who take all sizes of lobsters will benefit through increased catches and therefore increased individual profits. However, restrictions on maximum and minimum size benefit the group as a whole (the population of lobsterers in Maine), as they protect the reproduction of the lobster population, and therefore prevent a 'tragedy of the commons' from taking place.

Waring and Acheson describe the passage of double gauge laws as follows: a law of this kind was initially proposed in 1905, but what they term 'between group differences' caused by varying ecological and economic environments prevented its passage. Then, during the 1920s and 1930s the lobster industry in Maine experienced a significant decline in catches, and competition from the Canadian lobster industry, which led to a third of lobster-fishers leaving the industry. Waring and Acheson describe this as a type of 'cultural natural selection'. According to their narrative, these crises contributed to the passage of the double gauge law in 1933. Its passage was aided by the work of prominent actors, such as the Commissioner of Sea and Shore Fisheries, who spread support amongst lobstermen and legislators. According to the authors, selection at the individual level and at the state level were in conflict, and this was the reason for the political deadlock, until a major statewide crisis allowed the law to pass, which they term "strong group-level selection" (p. 27).

This is a case where we could deny that cultural selection is an appropriate explanation at all. Without delving into this in depth, it seems that we could deny that there are groups here that are the right kind of cultural group for selection to take place (for example, we could question where states compete in the relevant sense). However, charitably, we can find a way to construe it as cultural selection on both the individual and group level: individuals who adopted the double gauge rule prior to the institution of the law would have been less successful than those who did not, and so the double gauge rule did not spread amongst individual lobsterers. Once crisis hit, the state of Maine had to adopt the double gauge law (perhaps copying it from neighbouring states, or Canadian provinces). ${ }^{10}$

Even so, the question of explanatory power seems highly relevant. Taking Waring and Acheson's description of the historical changes as accurate, we can construct an alternative account that captures these changes, without the selection machinery. Under a goal-directed agent account, the law did not pass when it was first proposed due to the varying interests of those who would have to support the law in

\footnotetext{
Footnote 9 (continued)

of cultural populations that can undergo selection in order to focus on the explanatory power of cultural selection. One core feature of cultural group selection, as with cultural selection more generally, is its capacity to explain 'design without a designer'.

10 If Maine did copy the law from another state or province, this could be construed as an instance of 'selective imitation', as described in Richerson et al. 2016. This is one type of cultural group selection where we can explain the spread of particular cultural traits through the imitation of successful groups.
} 
order to ensure its passage: many lobster fishermen and political actors representing their interests regarded the laws as simply limiting the profits they could make, and thereby harming the industry. The crisis in the 1920s and 1930s led to individuals changing their attitudes, as they realised that such conservation laws were necessary for the survival of their industry. Acheson himself, in previous work, characterises the passage of the law in this way. In an editorial piece for Anthropology News, he describes the attitudes of many lobster fishermen before the industry crisis as being happy to flout existing conservation rules, with many believing that breaking these rules would have little effect on the sustainability of the resource and therefore their livelihood. The extent of the crisis the industry subsequently faced had a causal role in changing these individual attitudes, meaning that lobster fishermen became increasingly convinced that conservation laws such as the double gauge law were critical for the survival of lobstering in Maine (Acheson 2010).

We can also include the role of powerful actors in such an account: Acheson (1997) describes Horatio Crie, the then Commissioner of Sea and Shore Fisheries, as rejecting a law that would have reduced the minimum size further, in the belief that this would cause the demise of the lobster population. He supported a double gauge law, seeing it as a political compromise and necessary for the longevity of the lobster industry. Although politicians in the Maine legislature were divided over this issue, with the help of a forceful speech given by Crie in support of the law, the law passed. Such an account explains cultural phenomena through the decision-making of individual agents, while still being able to accommodate institutional structures and cultural norms that constrain the decisions that individuals make.

I suggest that the goal-directed agent account is at least as explanatorily powerful as the cultural selection account here. The goal-directed agent account reduces our surprise at the explanandum (the passage of the law); it becomes significantly more expected. Perhaps a more thorough and nuanced historical account would reduce our surprise further. Regardless, a cultural selection account cannot reduce our surprise any further than the goal-directed agent account can.

\section{Cassava processing in South America}

Joseph Henrich, in his 2015 book The Secret of Our Success, identifies a class of cultural phenomena that are arguably underexplained by historical, anthropological, or sociological frameworks, and proposes that these should be a particular target of cultural selection explanations. The class of phenomena that Henrich identifies is those behaviours which are adaptive (in that they are beneficial to an individual or group), but, crucially, where individuals are perhaps not aware of why these behaviours are adaptive, would find it difficult to find out why these are adaptive, and have not designed them specifically to be so. Here, accounts which rely on individual agency would struggle to explain the emergence, spread and persistence of these behaviours.

One example that Henrich uses is the consumption of cassava. Some varieties of cassava contain dangerous levels of cyanide, which if eaten without detoxification, could result in chronic health problems, such as goiter and neurological issues. 
However, communities in South America which rely on cassava as an important part of their diet, such as the Tukanoans, have a multi-step processing technique that occurs over several days and results in detoxified and safe to eat cassava. Henrich argues that any one individual would have difficulty working out this technique by themselves, and therefore individual learning fails to explain this adaptive behaviour. This is because the poisoning is slow, and so it would be difficult to figure out the connection between eating cassava and experiencing health issues. Additionally, the cassava stops being bitter before it stops being toxic, and therefore individuals who are relying on the signal of bitterness would continue to ingest unsafe levels of cyanide.

The steps in this procedure are 'causally opaque': individuals cannot easily infer the functions or importance of the behaviours they are carrying out. His explanation of the spread of this adaptive behaviour is based on social learning biases, including the unconscious tendency of people to imitate the behaviour of more successful, prestigious, or healthier members of their community. Over time, this behaviour spreads through a cultural selection process.

If we apply our strategy in this case, comparing Henrich's account with a goaldirected agent account, we are left with a very different result from the previous case. It is hard to see how a goal-directed agent account can explain why an individual carries out this time-consuming technique. A goal-directed agent account could likely explain certain aspects: the processing and consumption of cassava broadly could be understood with reference to an individual's goal of being fed. However, the key aspect of the phenomena (indeed, the most intriguing aspect), the multistep technique that has an adaptive benefit and yet no easily discernible connection between the technique and the benefit, are left 'surprising'. In contrast, the cultural selection explanation is clearly more powerful: social learning biases can drive the copying of adaptive traits, rendering this behaviour less surprising, or more expected. $^{1112}$

\footnotetext{
${ }^{11}$ This particular example has been called in question, with Mercier and Morin (2019) arguing that the ethnographic evidence suggests that the Tukanoans complete the final steps of cassava processing after it has stopped being bitter because they prefer the taste and texture after these steps. However, if we take Henrich's account of the behaviours at face value, this at least gives us an example of how a cultural selection explanation might be powerful.

${ }^{12}$ It is interesting to note that this stands out as a particularly persuasive example from Henrich's book. Of the other cases that he draws on from small-scale societies, some have a similar structure, where an adaptive technique is causally opaque. Others do not: one example concerns groups in New Guinea which raise pigs. The success of a group depends in part on the group's ability to raise large numbers of pigs. Henrich relays the work of anthropologist David Boyd who was living with one of these groups when the senior members decided to adopt the pig raising practices of another group, which was large, successful, and known for their pig production. These pig raising practices include not killing pigs who break into others' gardens, no longer sending pigs to other villages, and feeding the pigs more food. Henrich characterises this as intergroup competition via prestige-biased transmission. It is unclear to me that there is convincing causal opacity here: the link between not killing pigs, not sending them away, or taking better care of them and having more pigs seems relatively easy to work out.
} 


\section{Objections}

Here I will address two possible objections: firstly, that selection models are operating on a different level of explanation, and therefore the presence of successful intention-based explanations is not a reason to discount the application of cultural selection in a given case. Secondly, that cultural selection could still be a useful heuristic, and therefore could productively guide research.

\section{Are selection models operating at a different level of explanation?}

One might argue that a cultural selection account and a goal-directed agent account are operating at different levels, and that therefore this contrastive strategy is misplaced. This objection can be made clearer by considering a biological analogy. Let us take the classic example of Darwin's finches, where Darwin observed differences in the dimensions of the beaks of finches living on different islands of the Galapagos archipelago. Evolution by natural selection can explain these differences in beak shape through adaptation to the different available food sources: individuals with beak shapes that allowed them to more effectively utilise food resources were selected for, surviving and reproducing more than individuals with less welladapted beaks. However, we could also offer an explanation on the level of the lives of individual birds. We could track the birth and death of each individual finch, and describe in detail, and perhaps with agential terms, how they lived their life and how many offspring each finch had. This would give us the same information as in the selection explanation, as we would have tracked differential survival and offspring production, although without the overarching framework. We could therefore perhaps argue that there is no need to invoke selection here, as a life histories account also provides a successful explanation. However, this would be an odd argument to make in this biological case, when the explanatory power of natural selection seems so clear.

To apply this analogy to cultural selection, we could imagine that a historical account of cultural change might trace individual actors in great detail, whereas a cultural selection account would pick out particular types of causal processes. The cultural selection account may rely on the historical account in the sense that individual actions are driving the change, in the same way that a natural selection account relies on the life histories of individual organisms. Here, we might be tempted to think that a cultural selection account would be still be useful and powerfully explanatory, because by abstracting away from details of the lives of particular individuals we can gain some insight into what is driving changes in the population as a whole.

One way of understanding this objection is to think about it in terms of the difference between 'population thinking' and either a goal-directed agent approach, or historical or social scientific approaches. The potential for 'population thinking' to generate novel insights could provide a motivation for applying cultural selection models to particular phenomena, even if successful historical, social scientific, or 
anthropological explanations exist for these phenomena. In contrast to, for example, ethnographic work, which attends to the particulars of individual experiences, a 'population thinking' approach abstracts away from individuals and instead analyses the population-level consequences of the interactions of individuals with particular dispositions. Elliott Sober (1991, p. 492), in his analysis of the models of cultural evolution found in Cavalli-Sforza and Feldman (1981) and Boyd and Richerson (1985), suggests that the usefulness of these models to the "day-to-day research of social scientists" depends on how good social scientists are at "intuitive population thinking". Sober argues that social scientists are often primarily interested in the sources of particular ideas or norms, whilst cultural evolutionists are typically interested in the transmission patterns of these norms. However, social scientists do make qualitative assumptions about the transmission systems at work, and Sober claims that the cultural selection approach would impact social science work to the extent to which cultural selection models undermine these qualitative assumptions. Boyd and Richerson's (2005, p. 97) response, as highlighted by Lewens (2018), emphasises that we are not all good intuitive population thinkers. Therefore, we would expect the adoption of the 'population thinking' perspective to generate unexpected insights, that we would not have arrived at without this approach, motivating the development of cultural selection frameworks.

However, it is possible to accept the value of 'population thinking' without adopting cultural selection as an explanation for cultural phenomena. Lewens (2010, 2015) suggests that we can distinguish 'population thinking' (or kinetic theories of culture) from selection-based approaches. For example, a 'population thinking' approach could consider the population-level consequences that arise from a group of individuals with a particular social learning bias, such as the predisposition to adopt the most common cultural variant within their group (conformist bias). This may result in interesting and unintuitive results, such as the reduced ability for new cultural variants to spread throughout a group. We can understand this without invoking selection machinery here. This separation is clearly evident when considering the work of those in the 'cultural attractor' school of cultural evolution, such as Dan Sperber and Nicolas Claidière. These authors are often critical of cultural selection approaches (Sperber and Claidière 2006), and yet their own work is dependent upon 'population thinking', where this means understanding a cultural system as a "population of relatively autonomous items of different types with the frequency of types changing over time" (Claidière et al. 2014). Therefore, we need explicit motivation for applying cultural selection in particular, rather than adopting a general 'population thinking' perspective. The claim that cultural selection explanations can have equal explanatory power to goal-directed agent explanations is compatible with the possibility that a 'population thinking' approach could have value in these cases, although I will not suggest what this might look like or argue for this claim.

As I have argued in section 'Explaining 'Design without a Designer', the key motivator for cultural selection approaches appears to be the explanation of 'cultural adaptations', and therefore 'design without a designer'. If we accept this motivation, it seems that we can understand both cultural selection and goal-directed agent accounts to be at the same level and ultimately incompatible, rather than complementary. This is because, as I have argued, cultural selection should be able to explain 'design without 
a designer'. If a goal-directed agent account can explain the 'design', we have found a designer.

\section{Can selection be a useful heuristic?}

Another possible objection to the strategy I have outlined is that cultural selection could nevertheless provide a useful heuristic, even if it does not provide explanatory power over a goal-directed agent account in a particular domain. One potential benefit of using cultural selection frameworks could be the ability to ask certain kinds of questions: cultural selection can be seen as a heuristic, intended to generate hypotheses and structure empirical research. Cultural selection may therefore sometimes be a useful tool to structure and guide research, through changing the way that we approach phenomena and the kinds of phenomena that are the subjects of study.

I accept we cannot rule this out, and therefore deploying my comparative strategy and finding the cultural selection explanation lacking does not provide a watertight reason for dismissing cultural selection in a given case or domain. However, there is reason for caution here.

Northcott and Alexandrova (2015), in their critical examination of the explanatory power of the Prisoners' Dilemma in the social sciences, consider the possibility that the Prisoners' Dilemma has heuristic value, even if it has limited explanatory payoffs. They focus on one of the most well-known cases of the application of the Prisoners' Dilemma to real-world phenomena, Robert Axelrod's (1984) work on the 'live-and-letlive' system of informal truces that arose in World War I trenches. They argue that the reliance on pre-existing historical work shows that Prisoners' Dilemma-like thinking was already present in our analysis, and therefore did not lead to any causal explanations we did not already possess. We can see a similar issue arising in the work of Waring and Acheson on lobstering in Maine: the reliance on historical or anthropological work to drive the explanations seems to tell against the heuristic value of the cultural selection approach, as cultural selection is not generating any new questions that were not already guiding research into these phenomena.

Additionally, we must be wary of using selection as a heuristic when there are significant disanalogies between the structure of the phenomena in question and a selective process. This comes back to the question of whether cultural selection can provide an explanation at all. If cultural selection can explain, but only weakly, we might still see its value as a heuristic (although this value has to be demonstrated). If cultural selection, for reasons relating to the general debates about cultural systems outlined in section 'Explaining 'Design without a Designer', or because of features of a particular domain, cannot function as an explanation in the first place, then using selection as a heuristic might in fact lead us astray, unhelpfully distorting our understanding of cultural phenomena. 


\section{Conclusion}

Cultural selection is a growing research programme, that promises to explain a wide range of cultural phenomena, ranging from prosocial tendencies that emerged in our distant past to the current behaviour of firms in a marketplace. However, arguments for cultural selection have often relied on the Lewontin conditions, only demonstrating that selection models can be applied to culture, without demonstrating the aptness of the explanation or the explanatory payoffs that arises from their application. This has further importance when considering the claims made regarding practical benefits of employing cultural selection models in the context of innovation and sustainability science.

I have suggested that, although the debate around the analogy between cultural and biological systems has generated interesting and important insights, more attention needs to be paid to the explanatory power of cultural selection. I have argued that one core feature of cultural selection explanations is the capacity to explain 'design without a designer': this ability is a key component of their explanatory power. In order to evaluate that explanatory power, I have introduced the strategy of comparing cultural selection accounts with goal-directed agent accounts.

I have then drawn on case studies to illustrate how this strategy might usefully be deployed to separate out powerful cultural explanations from weaker ones. The case of lobster fishing in Maine has provided a clear demonstration of how selection explanations can be applied to phenomena in a way that is explanatorily weak. In contrast, the case of cassava processing by the Tukanoans provides an example of when cultural selection does appear to be a powerful explanation in comparison with a goal-directed agent explanation.

This conceptual strategy is intended to be a first step in assessing where and when cultural selection might provide a powerful explanation. Further work will then be needed to compare cultural selection accounts to more nuanced or detailed alternatives. Given that we are surrounded with a wealth of frameworks for understanding culture, this comparative approach is necessary for a rigorous evaluation of cultural selection.

Acknowledgements I am grateful to Tim Lewens, Anna Alexandrova, Andrew Buskell, Raphael Scholl, William Wong, Tyler Brunet, Adrian Currie, and Joseph Wu, as well as audiences at EPSA 2019 and the Human Success: Evolutionary Origins and Ethical Implications conferences for their helpful feedback and suggestions. I am also thankful for the comments of the reviewers, which have greatly improved this manuscript.

Funding This work was supported by the Arts and Humanities Research Council, Cambridge AHRC DTP Studentship.

Open Access This article is licensed under a Creative Commons Attribution 4.0 International License, which permits use, sharing, adaptation, distribution and reproduction in any medium or format, as long as you give appropriate credit to the original author(s) and the source, provide a link to the Creative Commons licence, and indicate if changes were made. The images or other third party material in this article are included in the article's Creative Commons licence, unless indicated otherwise in a credit line to the material. If material is not included in the article's Creative Commons licence and your intended use is not permitted by statutory regulation or exceeds the permitted use, you will need to obtain permission 
directly from the copyright holder. To view a copy of this licence, visit http://creativecommons.org/licen ses/by/4.0/.

\section{References}

Acerbi A, Mesoudi A (2015) If we are all cultural Darwinians what's the fuss about? Clarifying recent disagreements in the field of cultural evolution. Biol Philos 30(4):481-503

Acerbi A, Tehrani JJ (2018) Did Einstein really say that? Testing content versus context in the cultural selection of quotations. J Cogn Cult 18(3-4):293-311

Acheson JM (1997) The politics of managing the Maine lobster industry: 1860 to the present. Hum Ecol 25(1):3-27

Acheson JM (2010) Failure and success in fisheries management in the Gulf of maine. Anthropol News 51(7):25-25

Ariew A, Ernst Z (2009) What fitness can't be. Erkenntnis 71(3):289

Axelrod R (1984) The evolution of cooperation. Basic Books, New York

Beddoe R, Costanza R, Farley J, Garza E, Kent J, Kubiszewski I, Martinez L, McCowen T, Murphy K, Myers N, Ogden Z (2009) Overcoming systemic roadblocks to sustainability: the evolutionary redesign of worldviews, institutions, and technologies. Proc Natl Acad Sci 106(8):2483-2489

Birch, J. (2017). The philosophy of social evolution. Oxford University Press.

Boyd R, Richerson PJ (1985) Culture and the Evolutionary Process. Chicago: University of Chicago Press.

Boyd R, Richerson PJ (2002) Group beneficial norms can spread rapidly in a structured population. J Theor Biol 215(3):287-296

Boyd, R, Richerson PJ (2009) Voting with your feet: payoff biased migration and the evolution of group beneficial behavior. J Theor Biol 257(2):331-339

Boyd R, Richerson PJ, Henrich J (2011) Rapid cultural adaptation can facilitate the evolution of largescale cooperation. Behav Ecol Sociobiol 65(3):431-444

Cavalli-Sforza LL, Feldman MW (1981) Cultural transmission and evolution: a quantitative approach. Princeton University Press, Princeton

Chellappoo A (2021) Rethinking prestige bias. Synthese 198(9):8191-8212. https://doi.org/10.1007/ s11229-020-02565-8

Claidière N, Amedon GK-K, André J-B, Kirby S, Smith K, Sperber D, Fagot J (2018) Convergent transformation and selection in cultural evolution. Evol Hum Behav 39(2):191-202

Claidière N, Scott-Phillips TC, Sperber D (2014) How Darwinian is cultural evolution? Philosophic Trans Royal Soc B: Biol Sci 369(1642):20130368

Clarke E, Heyes C (2017) The swashbuckling anthropologist: henrich on the secret of our success. Biol Philos 32(2):289-305

Crozier GKD (2008) Reconsidering cultural selection theory. Br J Philos Sci 59(3):455-479

Dawkins R (1982) The extended phenotype: the long reach of the gene. Oxford University Press, Oxford

El Mouden C, André J-B, Morin O, Nettle D (2014) Cultural transmission and the evolution of human behaviour: a general approach based on the Price equation. J Evol Biol 27(2):231-241. https://doi. org/10.1111/jeb.12296

Elster J (1984) Ulysses and the sirens: studies in rationality and irrationality, Revised. Cambridge University Press, Cambridge

Godfrey-Smith P (2009) Darwinian populations and natural selection. Oxford University Press, Oxford

Godfrey-Smith P (2012) Darwinism and cultural change. Philosophic Trans Royal Soc London B: Biol Sci 367(1599):2160-2170

Henrich J (2015) The secret of our success: how culture is driving human evolution, domesticating our species, and making us smarter. Princeton University Press, Princeton

Henrich J, Boyd R, Richerson PJ (2008) Five misunderstandings about cultural evolution. Hum Nat 19(2):119-137

Henrich J, Henrich N (2010) The evolution of cultural adaptations: fijian food taboos protect against dangerous marine toxins. Proceed Royal Soc B: Biol Sci 277(1701):3715-3724

Kitcher P (1981) Explanatory unification. Philosophy Sci 48(4):507-531 
Kline MA, Waring TM, Salerno J (2018) Designing cultural multilevel selection research for sustainability science. Sustain Sci 13(1):9-19

Kronfeldner ME (2007) Is cultural evolution Lamarckian? Biol Philos 22(4):493-512

Laland KN, Sterelny K (2006) Perspective: seven reasons (not) to neglect niche construction. Evolution 60(9):1751-1762

Lewens T (2010) Natural selection then and now. Biol Rev 85(4):829-835

Lewens T (2015) Cultural evolution: conceptual challenges. Oxford University Press, Oxford

Lewens T (2018) Cultural evolution. In Edward N. Zalta (ed.) The Stanford Encyclopedia of Philosophy (Summer 2020 Edition). https://plato.stanford.edu/archives/sum2020/entries/evolution-cultural/

Lewontin RC (1970) The units of selection. Annu Rev Ecol Syst 1:1-18

Lipo CP, Madsen M (2001) Neutrality,"style", and drift: building methods for studying cultural transmission in the archaeological record. In: Hurt T, Rakita G (Eds) Style and function: conceptual issues in evolutionary archaeology. Bergin and Garvey, Westport, Connecticut.

McElreath R, Boyd R, Richerson P (2003) Shared norms and the evolution of ethnic markers. Curr Anthropol 44(1):122-130

Mercier H, Morin O (2019). Blind imitation or a matter of taste? [Blog post]. http://cognitionandculture. net/blogs/hugo-mercier/a-matter-of-taste/. Accessed 7 June 2019

Mesoudi A, Whiten A, Laland KN (2004) Perspective: Is human cultural evolution Darwinian? Evidence reviewed from the perspective of the Origin of Species. Evolution 58(1):1-11

Mesoudi A, Whiten A, Laland KN (2006) Towards a unified science of cultural evolution. Behavioral and Brain Sciences 29(4):329-347

Morin O (2018) Did social cognition evolve by cultural group selection? Mind Lang 34(4):530-539

Muthukrishna M, Henrich J (2019) A problem in theory. Nat Hum Behav 3(3):221-229

Neander K (1995) Pruning the tree of life. Br J Philosophy Sci 467:59-80

Nettle D (2020) Selection, adaptation, inheritance and design in human culture: the view from the Price equation. Philos Trans R Soc B 375(1797):20190358

Norenzayan A, Shariff AF, Gervais WM, Willard AK, McNamara RA, Slingerland E, Henrich J (2016) The cultural evolution of prosocial religions. Behav Brain Sci 39

Northcott R, Alexandrova A (2015) Prisoner's Dilemma doesn't explain much. In: Peterson M (ed) The Prisoner's Dilemma. Cambridge University Press, Cambridge, pp 64-84

Okasha S (2006) Evolution and the levels of selection. Oxford University Press, Oxford

Palmer CT, Fredrickson BE, Tilley CF (1997) Categories and gatherings: group selection and the mythology of cultural anthropology. Evol Hum Behav 18(5):291-308

Papale F (2020) Evolution by means of natural selection without reproduction: revamping Lewontin's account. Synthese 1-27

Pinker S (2012) The false allure of group selection. [Online Essay]. https://www.edge.org/conversation/ steven_pinker-the-false-allure-of-group-selection. Accessed 1 Feb 2021

Ramsey G, De Block A (2017) Is cultural fitness hopelessly confused? Br J Philos Sci 68(2):305-328

Richerson P, Boyd R (2005) Not by genes alone: How culture transformed human evolution. University of Chicago Press, Chicago

Richerson PJ, Boyd R (2005) Not by genes alone: how culture transformed human evolution. University of Chicago press

Richerson P, Baldini R, Bell AV, Demps K, Frost K, Hillis V, Mathew S, Newton EK, Naar N, Newson L, Ross C (2016) Cultural group selection plays an essential role in explaining human cooperation: a sketch of the evidence. Behav Brain Sci 39

Shennan S (2020) Style, function and cultural transmission. In: Groucutt H (ed) Culture history and convergent evolution. Vertebrate paleobiology and paleoanthropology. Springer, Cham

Schupbach JN, Sprenger J (2011) The logic of explanatory power. Philosophy Sci 78(1):105-127

Scott-Phillips TC, Laland KN, Shuker DM, Dickins TE, West SA (2014) The niche construction perspective: a critical appraisal. Evolution 68(5):1231-1243

Smith D (2020) Cultural group selection and human cooperation: a conceptual and empirical review. Evol Human Sci 2

Sober E (1991) Models of Cultural Evolution. In: Griffiths P (ed) Trees of Life: Essays in the Philosophy of Biology. Kluwer, Dordrecht, pp 477-492

Sperber D, Claidière N (2006) Why modeling cultural evolution is still such a challenge. Biol Theory 1(1):20

Stegenga J (2014) Population pluralism and natural selection. Br J Philos Sci 67(1):1-29 
Uller T, Helanterä H (2017) Niche construction and conceptual change in evolutionary biology. Br J Philos Sci 70(2):351-375

Waring T, Acheson J (2018) Evidence of cultural group selection in territorial lobstering in Maine. Sustain Sci 13(1):21-34

Wertz AE, Moya C (2019) Pathways to cognitive design. Behav Proc 161:73-86

Wimsatt WC (1999) Genes, memes, and cultural heredity. Biol Philos 14(2):279-310

Woodward J (2005) Making things happen: a theory of causal explanation. Oxford University Press, Oxford

Woodward J (2010) Causation in biology: stability, specificity, and the choice of levels of explanation. Biol Philos 25(3):287-318

Wray KB (2002) Social selection, agents' intentions, and functional explanation. Analyse \& Kritik 24(1):72-86

Zefferman MR, Mathew S (2015) An evolutionary theory of large- scale human warfare: group- structured cultural selection. Evol Anthropol 24(2):50-61

Publisher's Note Springer Nature remains neutral with regard to jurisdictional claims in published maps and institutional affiliations. 